\title{
TO LANGUAGE TEACHERS
}

THIS BOOK IS also directed to graduate students in TESOL and applied linguistics as well as language teachers who may wish to learn more about how to implement corpus activities in the classroom. I have often spoken with graduate students and language teachers who have said, 'I'm interested in doing corpus activities, but I'm not sure how,' or, 'I want to do corpus activities in my class, but I don't have time.' This book has emerged from these very statements. As a composition teacher in a first-year writing programme, I understand the challenge of balancing attention to rhetoric, process, and documentation while also trying to address the language needs of second language learners. The activities in this text attempt to find a happy medium between these various concerns. Many of the tutorials in this book were developed in response to particular classroom realities such as a need to develop my students' abilities to revise papers or improve word choice. Thus, the activities were initially created to solve particular needs of my students. Rather than consistently commenting on essays 'Avoid this ...' or 'You should do that...', I have developed a number of targeted corpus searches for students. These searches are designed to produce 'Aha!' moments that will help students make more effective choices in their own writing.

In this text, you will learn how to help students complete searches to revise their essays, create wordlists for vocabulary study, investigate collocates to improve word choice, and see how corpus searches can stimulate engaging classroom conversation and even facilitate student research projects. Throughout the text, there are sections titled 'Your Turn' that ask readers to pursue their own searches and reach their own conclusions about language use.

I hope those earlier stated concerns of 'How?' and 'When?' will be mitigated as the tutorials help you see how you can design your own activities and the learning outcomes that may be achieved while also providing you with classroom-ready activities that you can begin implementing in your classrooms immediately. 
